\title{
Glutamine Supplementation and Deprivation: Effect on Artificially Reared Rat Small Intestinal Morphology
}

\author{
BRADLEY POTSIC, NICOLETTE HOLLIDAY, PAT LEWIS, DONALD SAMUELSON, \\ VINCENT DEMARCO, AND JOSEF NEU \\ Division of Neonatology, Department of Pediatrics, J. Hillis Miller Health Center, University of Florida \\ College of Medicine, Gainesville, Florida 32610, U.S.A.
}

\begin{abstract}
The mechanisms of how glutamine benefits critically ill patients have not been established. The purpose of this study was to determine the effects of dietary and endogenously produced glutamine on small intestinal morphology using light and transmission electron microscopy in artificially reared rat pups. It was hypothesized that deprivation of dietary glutamine leads to intestinal disease that is exacerbated by inhibition of glutamine synthetase by methionine sulfoximine (MS). Rat pups were placed into five different test groups: The first was a reference group that was reared by their mother. The other four groups were reared artificially and received a 10\% Travasol amino acid solution at $5 \mathrm{~g} / \mathrm{kg}$ per day, which does not contain glutamine, added to a mixture containing carbohydrates, lipids, and vitamins. This dose was chosen because it represents an approximation of the amount of glutamine these rats would be receiving in a normal rat diet (approximately $40 \mathrm{~g} / \mathrm{kg}$ per day total protein, 10 to $15 \%$ of which is glutamine + glutamate). The glutamine was manipulated by adding glutamine (Q) or MS or both. The four groups were as follows: $\mathrm{MS}-\mathrm{Q}-, \mathrm{MS}-\mathrm{Q}+, \mathrm{MS}+\mathrm{Q}-$, and $\mathrm{MS}+\mathrm{Q}+$. Light microscopy revealed the greatest blunting of villus height in the ileum of rats from the MS+Q- group when compared with the MS-Q+ group $(123 \pm 48.9 \mu$ m versus 207
\end{abstract}

\section{ABSTRACT}

$\pm 36 \mu \mathrm{m}, p<0.05)$. The other two groups exhibited intermediate villus heights, but all were shorter than the villi from the mother-reared animals. The number of villi per unit length of bowel was also lowest in the animals that were treated with MS and not provided with dietary glutamine. Transmission electron microscopy demonstrated breakdown of the epithelial junctions in the glutamine-deprived and glutamine synthetase-inhibited intestines. Glutamine-deprived animals also displayed sloughing of microvilli, decreased actin cores, and degeneration of the terminal web. In summary, these studies support the hypothesis that glutamine is involved with maintenance of intestinal epithelial integrity. (Pediatr Res 52: 430-436, 2002)
Q, glutamine
Abbreviations
MS, methionine sulfoximine
GS, glutamine synthetase
TPN, total parenteral nutrition
VLBW, very low birthweight
H\&E, hematoxylin and eosin
TEM, transmission electron microscopy

Previous studies have found that glutamine supplementation significantly decreases morbidity among critically ill patients. Adult bone marrow transplant recipients who received glutamine i.v. had significant decreases in length of hospital stay, hospital-acquired sepsis, and hospital costs $(1,2)$. Studies in VLBW infants found that glutamine supplementation resulted in decreased morbidity and hospital costs (3-5). Additionally, studies in rodents found that glutamine supplementation provides protection against sepsis and subsequent distal organ

Received July 4, 2001; accepted January 15, 2002.

Correspondence and reprint requests: Josef Neu, M.D., Department of Pediatrics, Box 100296, J Hillis Miller Health Center, University of Florida College of Medicine, Gainesville, FL 32610, U.S.A.; e-mail address: neuj@peds.ufl.edu

Supported by NIH R01 HD38954.

DOI: 10.1203/01.PDR.0000025347.92120.02 damage $(6,7)$. The mechanisms of how glutamine acts to promote these beneficial effects are not known. However, there is evidence that its effect on intestinal mucosal integrity may play a role. The addition of glutamine to TPN has been demonstrated to maintain the integrity of small intestinal mucosa, therefore, preventing the atrophy typically noted with TPN (8). In a previous study of multiple animal species, administration of glutaminase decreased plasma glutamine levels to almost undetectable levels (9). This resulted in mild villous atrophy, mucosal ulcerations, and intestinal necrosis.

In addition to the small intestine being a major site of utilization of glutamine derived from nonintestinal sources, there is evidence that the intestine also produces glutamine (10-13). The activity of GS increases just before weaning in the rat (11). The role of de novo synthesized glutamine in the 
small intestine is poorly understood. Studies using immunofluorescence labeling and in situ hybridization, designed to determine the localization of GS in small intestine, established that intestinal glutamine production primarily occurs in the crypt, a region of rapid cell proliferation (10). Studies using IEC-6 cells have demonstrated that inhibition of GS with MS, an inhibitor of GS (14), causes a significant decrease in proliferation that requires supraphysiologic concentrations of exogenous glutamine for reversal (15). Inhibition of GS in Caco-2 cells grown in transwell cultures results in decreased transepithelial resistance, opening of intercellular junctions, and decreased microvilli seen on TEM (16).

In this study we used light microscopy and TEM to determine the effects of both dietary and endogenously produced glutamine on small intestinal morphology. The hypothesis is that deprivation of dietary glutamine leads to intestinal pathologic changes that can be exacerbated by MS, an inhibitor of endogenous glutamine synthesis (14).

\section{METHODS}

The University of Florida Institutional Animal Care and Use Committee approved the following study.

Animal model. Thirty Sprague-Dawley (Zivic Miller Laboratories, Zelienople, PA, U.S.A.) infant rats were randomly assigned to four artificial feeding groups with six to seven rats per group. A mother-reared group $(n=3)$ was used as a reference. Artificial feeding using the rat infant "pup in the cup" model began on d 7 of life through feeding tubes constructed from 14-cm sections of polyethylene tubing that were inserted into the stomach, as described by Phillips et al. (17). This is a commonly used model in studies of developmental nutrition when it is important to manipulate nutritional composition in the absence of maternal feedings. The gastrostomy placement was performed under methoxyflurane anesthesia. Timer-controlled syringe pumps were connected to the feeding tubes and were set to feed the rats for the first $20 \mathrm{~min}$ of every hour at a weight-dependent flow rate. After a 1- to 2-d acclimation period during which they were fed with rat milk substitute (18), the protein component was changed from casein and whey to a $10 \%$ Travasol amino acid solution, which was infused at a dose of $5 \mathrm{~g} / \mathrm{kg}$ per day. This amino acid solution does not contain glutamine. This intake represents protein deprivation in these rats, that otherwise would require 30 to $40 \mathrm{~g} / \mathrm{kg}$ per day for normal growth (18). All the artificially fed groups received the same quantity of fat and carbohydrates. These pups were protein-deprived to partially mimic nutritional conditions in the Neonatal Intensive Care Unit, where VLBW infants are frequently not enterally fed for days to weeks and have their TPN amino acids slowly advanced over the first 1-2 wk of life.

MS, an irreversible GS inhibitor (14), was added to the Travasol-containing rat milk substitute at a concentration of 1 $\mathrm{mM}$ to inhibit the endogenous de novo synthesis of glutamine in the gastrointestinal tract. The glutamine and MS in the four treatment groups was manipulated by adding $2 \%$ glutamine $(\mathrm{wt} / \mathrm{vol}$ or $4 \mathrm{~g} / \mathrm{kg}$ per day) (Q+ group), adding MS (MS+ group), adding $\mathrm{MS}$ and glutamine (MS $+\mathrm{Q}+$ group), and not adding either (MS $-\mathrm{Q}-$ group) to the formula. At the end of the 4-d treatment period, the rat pups were euthanized with an overdose of sodium pentobarbital. The small intestine was removed and separated into three parts - the ileum, jejunum, and duodenum - and fixed for $24 \mathrm{~h}$ in $10 \%$ neutral buffered formalin.

Light microscopy. We focused the microscopy studies on the ileum because this is a region that is most highly susceptible to certain pathologic changes in VLBW infants (e.g. necrotizing enterocolitis and nonnecrotizing enterocolitisrelated perforations). Furthermore, pilot evaluations of the duodenum and jejunum did not suggest major differences. Formalin-fixed ileum samples were embedded in paraffin; 6- $\mu \mathrm{m}$ sections were cut using a 2030 Reichert-Jung paraffin microtome (Nusslock, Germany). The sections were then stained with a routine H\&E stain (19). Villus height and width were measured using a Nikon microscope (Universal Imaging Corp., Westchester, PA, U.S.A.) with an ocular micrometer without the examiner knowing the group assignment. The epithelial layer was measured at three points: two in the middle and one at the tip of the villus. This was done to assure the villus was not obliquely cut. Villus height was calculated by first measuring from the tip of the villus to the base of the tunica muscularis. Next, measurements were taken from the base of the villus to the base of the tunica muscularis on both sides of the villus; these values were then averaged. The averaged value from the bottom of the villus to the base of the tunica muscularis was then subtracted from the first measurement, thus giving the true villus height. Approximately 5 to 10 villi were measured for each H\&E-stained section; each rat had three to four stained sections (15-40 villi per rat). Villus height and corresponding villus width data were combined to analyze villus height-to-width ratios. Lastly, villus density was determined by measuring the length (in micrometers) of parts of the bowel followed by counting the number of villi within that length. The number of villi was then divided by a unit length of intestine, giving a measurement of villi per micrometer.

Transmission electron microscopy. One- to two-centimeter sections of the ileum from three to five representative rats from each treatment group were dissected and placed in chilled $2 \%$ glutaraldehyde $/ 0.1 \mathrm{M}$ sodium cacodylate fixative. After dehydration and embedding in araldite epoxy resin, $1-\mu \mathrm{m}$ sections were made using glass knives on a Reichert Ultracut $\mathrm{S}$ ultramicrotome, stained with $1 \%$ toluidine blue in $1 \%$ sodium borate (20), and examined with a light microscope. Target areas were selected for ultrastructural observations, and ultrathin sections (approximately $80 \mathrm{~nm}$ in thickness) were cut using a diamond knife and stained with saturated uranyl acetate and Reynold's lead citrate. Sections were viewed on a Hitachi H7000 TEM (Hitachi Software Engineering, Yokohama, Japan). General appearance and frequency of the microvilli were determined. The apical surfaces, frequency of junctional complexes, intercellular spaces, and cytoskeletal elements (terminal web and actin core of microvilli) were analyzed.

Statistical analysis. Sigmastat statistical software (SPSS Inc., Chicago, IL, U.S.A.) was used to analyze villus measurements. Villus height and villus height-to-width ratios were analyzed using an ANOVA to determine whether a significant 
difference was present among all treatment groups. Additionally, Bonferroni $t$ tests were performed for pairwise comparison of villus height, villus height-to-width ratios, and villus density among treatment groups.

\section{RESULTS}

\section{Animals}

Mortality in the individual groups was as follows: MS $-\mathrm{Q}-$, two of six died (33\%); MS $-\mathrm{Q}+$, one of six died $(16.7 \%)$; $\mathrm{MS}+\mathrm{Q}+$, two of six died (33.3\%); MS+Q-, two of seven died $(28.5 \%)$; and mother-reared, zero of three died $(0 \%)$. The motherreared animals gained $33 \%$ weight during the course of the study, whereas the artificially reared rats lost $13 \%$ without a difference in weight loss among the various artificially reared rat groups.

\section{Morphology and Epithelial Integrity}

Light microscopy. An ANOVA performed on the villus height data shown in Figure $1 A$ revealed statistically significant differences among the various treatment groups $(p \leq 0.001)$. Individual contrasts using a $t$ test with Bonferroni correction revealed that pups treated with MS, the GS inhibitor, and receiving no glutamine in their $\operatorname{diet}(\mathrm{MS}+\mathrm{Q}-)$ had significantly shorter $(p=0.02)$ villus height in comparison with glutamine-supplemented pups $(\mathrm{MS}-\mathrm{Q}+)$. Similarly, individual contrasts performed between mother-reared pups and each of the artificially reared treatment groups revealed that the mother-reared pups had significantly longer villi $(p<0.02)$.

An ANOVA of the villus height-to-width ratios (Fig. $1 B$ ) also revealed a significant difference among all five groups ( $p$ $=0.002)$. Individual contrasts of villus height-to-width ratios revealed the glutamine-supplemented $(\mathrm{MS}-\mathrm{Q}+$ ) group had a significantly larger ratio than the MS-treated group (MS $+\mathrm{Q}-$; $p=0.029$ ). In addition, the mother-reared infant rats had a significantly larger villus height-to-width ratio than MS-Qand $\mathrm{MS}+\mathrm{Q}-$ rat pups $(p<0.05)$.

Additionally, ANOVA of villus density (villi per micrometer; Fig. $1 C$ ) revealed a significant difference among the treatment groups $(p<0.001)$. Individual contrasts of villus density revealed the glutamine-supplemented group $(\mathrm{MS}-\mathrm{Q}+)$ had significantly greater villus density than the group that was given MS and not supplemented with glutamine (MS $+\mathrm{Q}-; p$ $<0.001)$. Similarly the MS $-\mathrm{Q}-$ group also had significantly greater villus density than MS $+\mathrm{Q}-$ group $(p<0.001)$. Furthermore, the mother-reared group had significantly greater villus density than both the $\mathrm{MS}+\mathrm{Q}+$ group and the $\mathrm{MS}+\mathrm{Q}-$ group.

Representative photos of H\&E-stained sections are shown in Figure 2. These show the effects of dietary glutamine deprivation in the presence or absence of MS. The villus morphology corresponds to the graphs in Figure 1. From these sections it is readily seen that the control group was the only group with fat globules in the epithelial cells, an effect we frequently see in normal, mother-fed rats. In addition to the villus blunting described by Figure 1, it is evident from these photos that the intervillus distance appears greatest in the animals treated with MS (Fig. 2, $C$ and $D$ ).
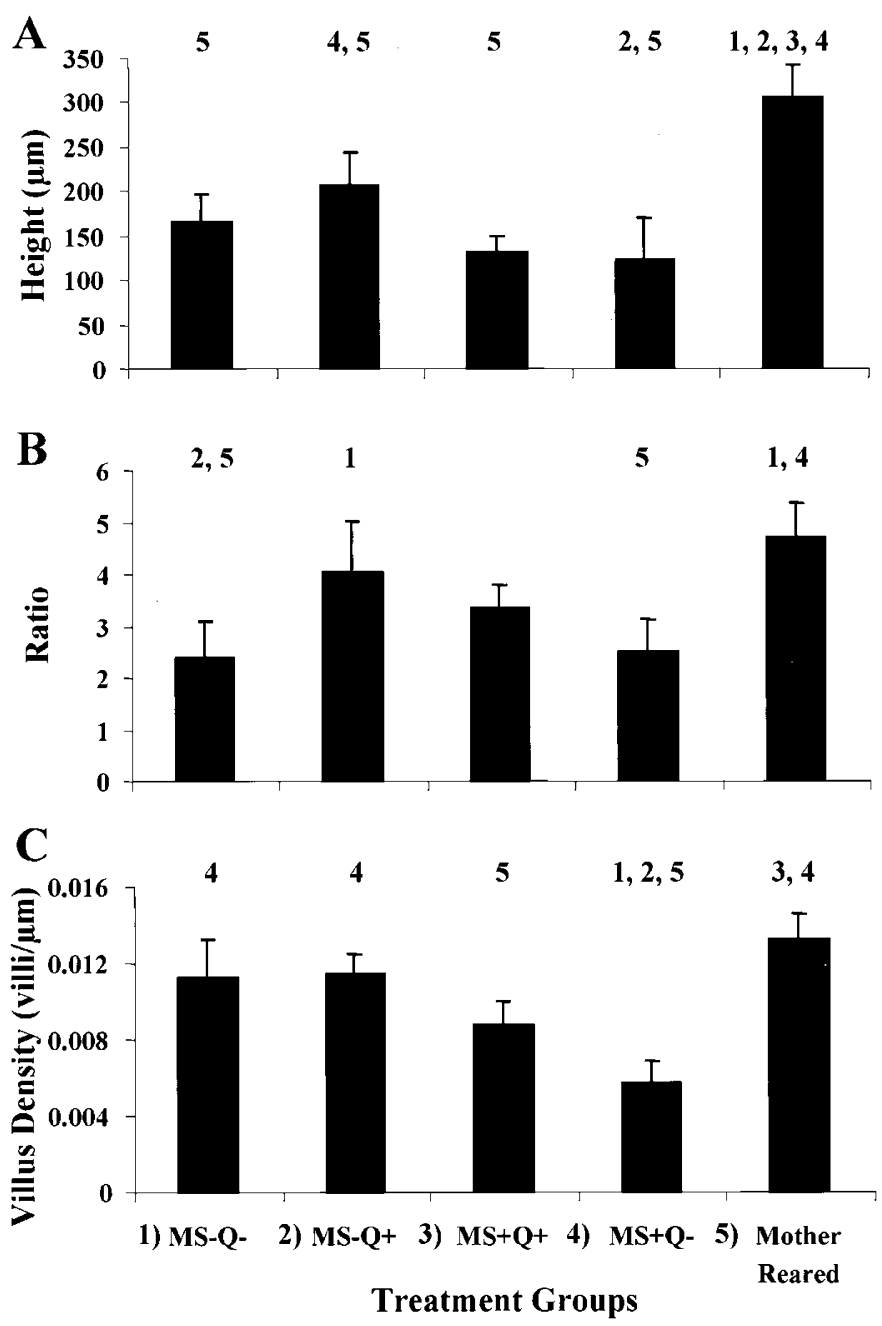

Figure 1. Bar graphs represent differences in villus height $(A)$, height:width ratios $(B)$, and villi density $(C)$ among different treatment groups (data reported as mean $\pm \mathrm{SD}$ ). The sample size of the animals that survived the study are represented by $n$. Numbers located above each bar represent statistically significant differences $(p<0.05)$ between that bar and the corresponding numbers of the treatment groups, which are labeled on the $x$ axis The groups are read from left to right.

Transmission electron microscopy. Figure 3 shows representative micrographs from the $\mathrm{MS}-\mathrm{Q}+$ and $\mathrm{MS}+\mathrm{Q}-$ groups, respectively. Figure $3 B$ reveals uneven and deteriorating microvilli that are sloughing in the $\mathrm{MS}+\mathrm{Q}-$ group. In addition to the microvillus sloughing, lack of actin cores, which normally are present within the microvilli, were observed in this group. Similar findings were found in the $\mathrm{MS}+\mathrm{Q}+$ group (not shown). The lack of actin core in the microvilli was more pronounced in the $\mathrm{MS}+\mathrm{Q}-$ group (denoted by arrow $L A C$ in Fig. $3 B$ ) than in the $\mathrm{MS}+\mathrm{Q}+$ group. In fact, most microvilli of the MS+Q- group tended to be flexible, making the entire length difficult to observe. The flexible nature of these microvilli corresponds to their lack of actin cores. The terminal web was also significantly less noticeable in the MS $+\mathrm{Q}-$ group in comparison with the $\mathrm{MS}-\mathrm{Q}-, \mathrm{MS}-\mathrm{Q}+$, and mother-reared rat pups. Furthermore, cell death, deteriorating intercellular junctions (denoted by arrow $D I J$ in Fig. $3 B$ ), splitting between cells, and in some 

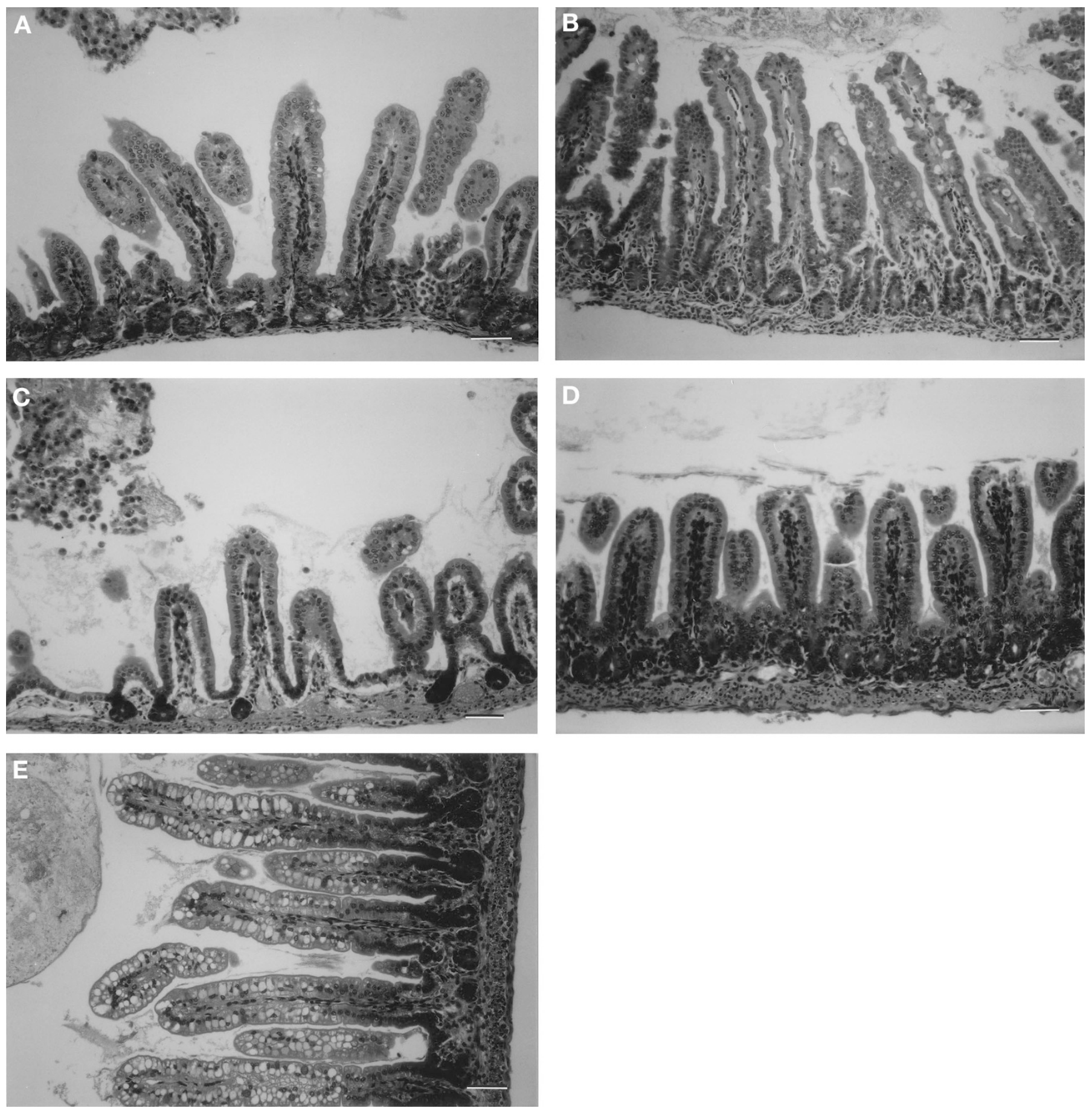

Figure 2. Representative H\&E-stained ileum sections of each treatment group at approximately $\times 930$ magnification (original magnification; $\times 200$ ) from which villus length and villus height-to-width ratios seen in Figure 1 were derived (please note: not every villus in the photos was measurable). Scale bars in each picture represent $50 \mu \mathrm{m}$. $\mathrm{MS}-\mathrm{Q}-(A), \mathrm{MS}-\mathrm{Q}+(B), \mathrm{MS}+\mathrm{Q}+(C)$, and $\mathrm{MS}+\mathrm{Q}-(D)$, and mothered-reared $(E)$.

cases numerous large intercellular spaces were also noted among the rats that received MS. Comparison of all five groups' tissue sections revealed less-developed junctional complexes in the MS+Q- and MS+Q+ treatment groups. In contrast, the tissue sections from the $\mathrm{MS}-\mathrm{Q}+$ (Fig. 3A), $\mathrm{MS}-\mathrm{Q}-$, and mother-reared groups appeared normal with tightly packed and even microvilli. The microvilli consisted of strong actin cores (denoted by arrow $A C$ in Fig. $3 A$ ) extending downward from the apical surface and often anchoring to the terminal web. The epithelial cells in the $\mathrm{MS}-\mathrm{Q}+$ group also displayed a higher degree of interdigitation than the other groups (denoted by arrow $I D$ in Fig. $3 A$ ). Numerous intact intercellular junctions, with noticeable zonula occludens, zonula adherens, and desmosomes, were present within the $\mathrm{MS}-\mathrm{Q}+$ group (denoted by arrow $T J$ in Fig. $3 A$ ), as well as the MS- - -, and mother-reared groups (not shown). 
A

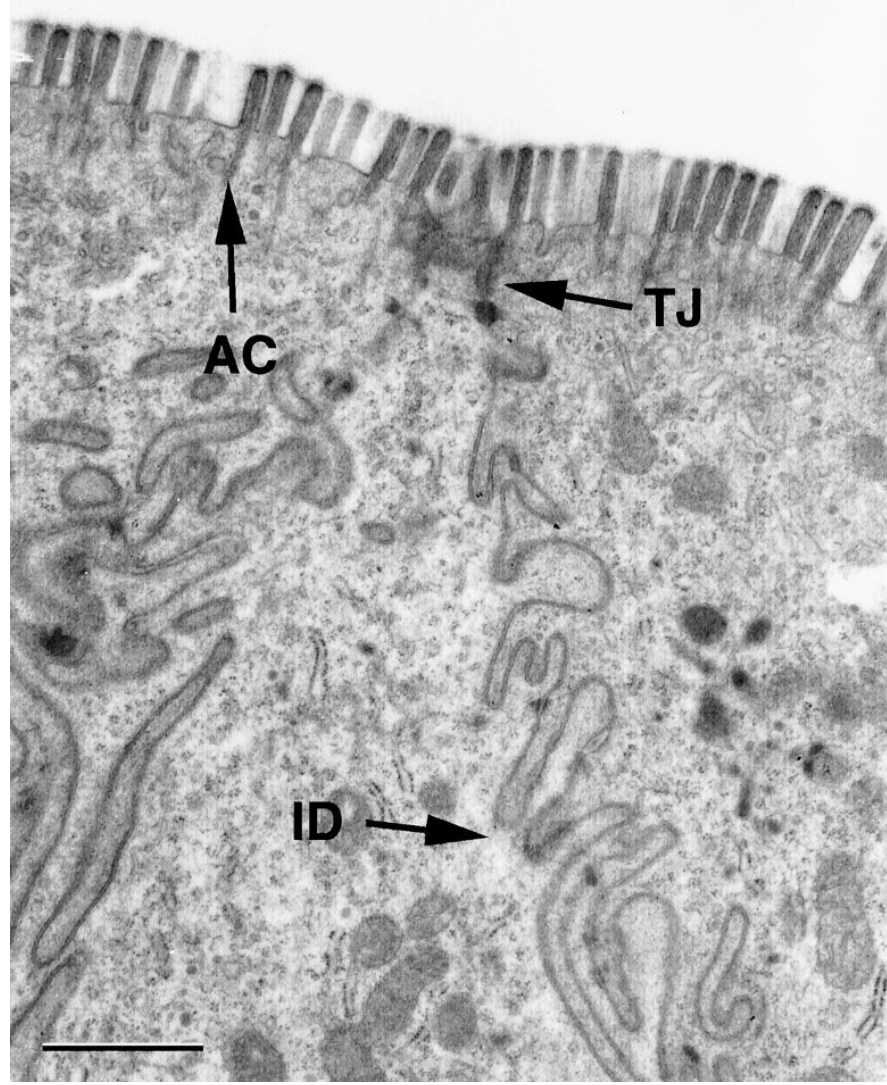

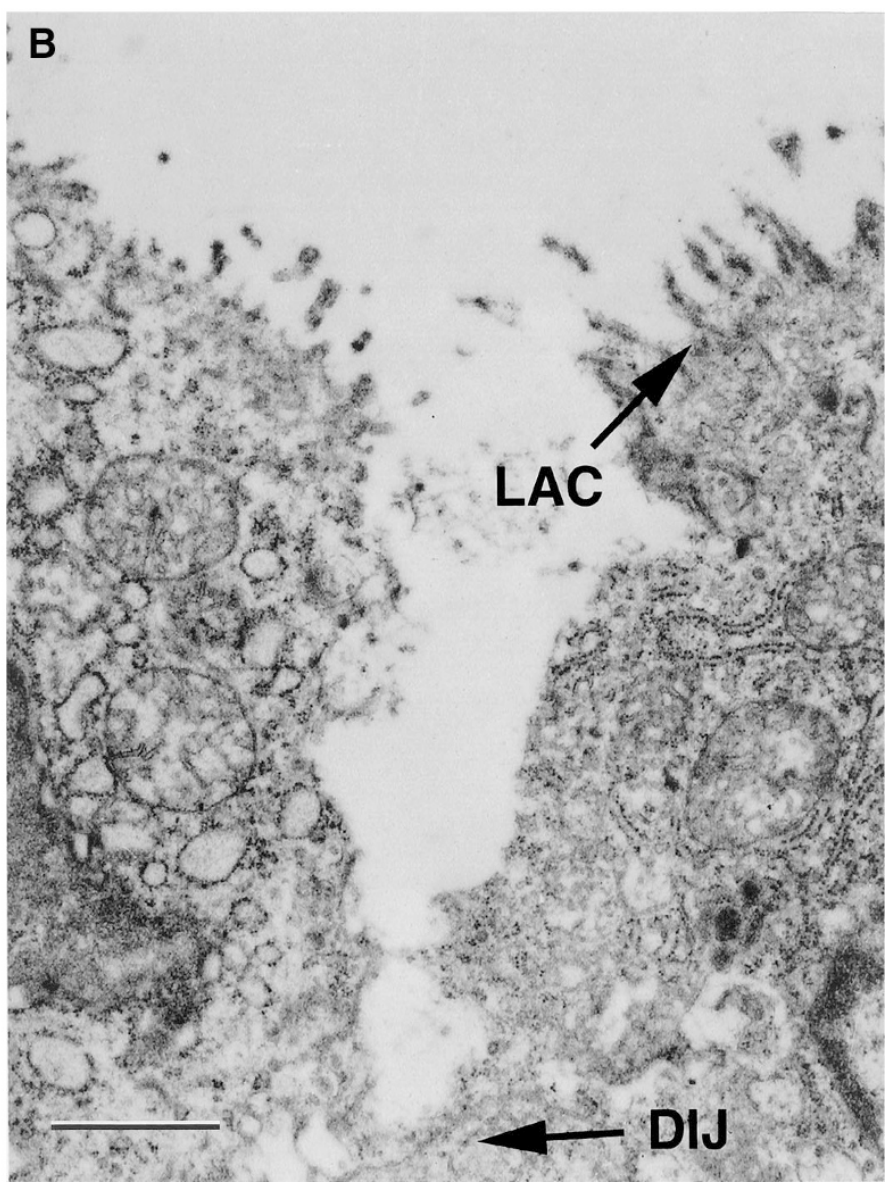

Figure 3. TEM photos of notable ultrastructural elements in the $\mathrm{MS}-\mathrm{Q}+$ group $(A)$ and $\mathrm{MS}+\mathrm{Q}-$ group $(B)$. The $\mathrm{MS}-\mathrm{Q}+$ group had a high degree of intercellular digitations (denoted by arrow $I D$ ). The MS $-\mathrm{Q}+$ group also had notable tight junctions (denoted by arrow $T J$ ) and other junctional complexes. In addition, the microvilli in the $\mathrm{MS}-\mathrm{Q}+$ group had prominent actin cores (denoted by arrow $A C$ ). The $\mathrm{MS}+\mathrm{Q}-$ group displayed microvilli deteriorating and sloughing off; microvilli remaining exhibited a lack of actin cores (denoted by arrow $L A C$ ). The MS $+\mathrm{Q}-$ group also showed evidence of cells pulling apart or deteriorating intercellular junctions (denoted by arrow $D I J$ ). Approximate magnification of images is $\times 20,000$. Scale bars in each picture represent $1 \mu \mathrm{m}$.

\section{DISCUSSION}

The significantly longer villi in the glutamine-supplemented group $(\mathrm{MS}-\mathrm{Q}+)$ and the significantly shorter villi in pups subjected to endogenous glutamine synthesis inhibition (MS+Q-) support our hypothesis that both dietary and endogenously synthesized glutamine promotes intestinal integrity. The villus height-to-width ratios provided another measurement for overall gut health. The glutamine-supplemented group not treated with MS had the largest height-to-width ratio of all the artificially fed groups, and that ratio was the most comparable to that of the mother-reared group. Qualitative comparisons revealed that the $\mathrm{MS}-\mathrm{Q}+$ group had the greatest villus density among all artificially reared treatment groups (Fig. 2B). This implies that glutamine increases the surface area for absorption of nutrients within the small intestine of infant rats that are concomitantly deprived of dietary protein. This was further supported in the findings of villus density inasmuch as the MS $-\mathrm{Q}+$ group had significantly greater villi density than $\mathrm{MS}+\mathrm{Q}-$. Of note, rats receiving MS that also received supplemental glutamine $(\mathrm{MS}+\mathrm{Q}+)$ only exhibited a minor "rescue" on the pathologic features of the small intestine caused by MS and glutamine deprivation (MS+Q-), suggesting that the benefits of dietary glutamine are optimized when enterocytes can normally synthesize glutamine. It is unknown whether or not this synergistic effect represents partitioning of exogenous and endogenous sources of glutamine carbon and nitrogen into downstream metabolites or whether the dietary supplementation that was given was insufficient.

The TEM results also supported the hypothesis. The glutamine-supplemented group ( $\mathrm{MS}-\mathrm{Q}+$ ) displayed a high degree of intercellular interdigitation, which is associated with increased intercellular adhesion, and enhancement of the epithelial barrier. The deprivation of endogenous glutamine by administration of MS resulted in the breakdown of the epithelial barrier. Among the two treatment groups that received MS, intercellular junctional complexes were less prevalent than those observed in the glutamine-supplemented, control, and mother-reared groups. Because the epithelial cells are more tightly attached at this junctional region than anywhere else on the cellular surface, a lack of these tight junctions could have significant detrimental effects on the integrity of the gut. The presence of large intercellular spaces, apparent splitting between epithelial cells, and cell death within the MS+Q- and $\mathrm{MS}+\mathrm{Q}+$ groups also supported our hypothesis that endogenous glutamine is important for the maintenance of the gut integrity. 
The endogenously glutamine-deprived infant rats also displayed significant degeneration of the apical surface including sloughing of microvilli. As a result, few microvilli remained on any given epithelial cell. Furthermore, these same infant rats also lacked the presence of distinguishable actin cores within the microvilli (Fig. 3B). Typically an actin core extends longitudinally down the middle of the microvillus and is anchored to the terminal web. This type of ultrastructure was not noted in these rats' tissue. Lastly, it was also difficult to discern the presence of terminal webs in the intestinal epithelial tissue of the $\mathrm{MS}+\mathrm{Q}-$ and $\mathrm{MS}+\mathrm{Q}+$ rats. Because the terminal web is connected to the zonula adherens, a component of the junctional complex, the lack of terminal webs correlates to the infrequency of intercellular junctional complexes. Moreover, the lack of actin cores among the few remaining microvilli correlates to the infrequency of terminal webs as these actin cores are known to attach to the terminal web. Actin cores, terminal webs, and zonula adherens are all either composed of cytoplasmic filaments or connected to these filaments. As a result, the lack of actin cores in microvilli, degradation of the terminal web, and few junctional complexes among the endogenously glutamine-deprived rat pups suggest that glutamine could play a role in maintaining a healthy cytoskeleton within cells. Because the cytoskeleton is related to the junctional complex, the healthy cytoskeleton that may be promoted by glutamine could be one mode by which glutamine enhances healthy small intestinal integrity and morphology. In summary, these results demonstrate the importance of dietary glutamine in maintaining the integrity of the small intestinal surface in the setting of limited neonatal nutrition.

Although it is likely that these findings are specific to glutamine, it is also possible that supplementation with other nutrients (amino acids, nucleotides) could result in similar outcomes. As previously stated the glutamine supplementation was in addition to a glutamine-free amino acid solution the pups were receiving. This solution was used because it was a readily available source to provide an amino acid mixture that is free of glutamine. In this experiment there were no isonitrogenous controls to balance an equal amount of amino acid intake among each group. As a result, the beneficial findings, which we ascribe to glutamine, could possibly be related to increased amino acid intake rather than being specific to glutamine supplementation. However, the use of isonitrogenous controls presents a dilemma as to what amino acid or group of amino acids should be used. Most have their own potentially beneficial or adverse effects. Because of this, we chose to examine the effects of glutamine supplementation versus no glutamine, as would be the case in clinical practice.

Previous studies point to the likelihood that breakdown of the intestinal mucosal barrier results in translocation of bacteria into the bloodstream (21-23). Evidence is accumulating that glutamine supplementation prevents mucosal breakdown and decreases intercellular hyperpermeability and subsequent translocation of bacteria (24-31). Cell culture studies from our laboratory have demonstrated that inhibition of endogenous glutamine synthesis decreases proliferation (15) and intercellular junctional integrity (examined at the TEM level) (16). Further preliminary in vivo studies have demonstrated in- creased mortality and bacterial translocation with GS inhibition (32).

The mechanism of protection provided by glutamine remains poorly understood. Because of the myriad of metabolic processes in which glutamine is involved, the mechanism is probably multifactorial. The importance of glutamine and glutamate to enterocytes for energy production has been established (33-35). Glutamine also acts to stimulate crypt cell proliferation via mitogen-activated protein kinases $(36,37)$ and is antiapoptotic via its interaction with glutamine-tRNA synthase, which in turn interacts with apoptosis signal-regulating kinases (38). Furthermore, the utilization of the amide-N of glutamine for nucleotides has also now been realized (35). Recent studies suggest that the amide- $\mathrm{N}$ of glutamine is also important for incorporation into hexosamines $(39-41)$. Hexosamines, as components of glycoproteins and amino sugars, are very important in maintaining absorptive (via glycosylated membrane digestive hydrolases) and gut barrier functions (via the surface mucin barrier and glycoproteins that form intercellular tight junctions). The fuzzy glycocalyx coat of the villi, consisting of a family of glycoproteins and proteoglycans (of which the hexosamines are a major carbohydrate component), protects the intestine from autodigestion by luminal enzymes and may also protect the rest of the body from bacterial invasion. The importance of glutamine for maintenance of the mucus and intercellular junctional barrier are supported by studies that have demonstrated the critical relationship between glutamine and the mucus lining of the small intestine in the rat (41). These studies demonstrated that alanyl-glutaminesupplemented TPN resulted in significant increases in goblet cells, glucosamine synthetase activity, and OD of mucus gel lining the intestine. This was associated with a decrease in permeability to FITC dextran.

There remains controversy about the relative benefits of glutamine for maintenance of intestinal morphology. Buchman (42) has questioned whether glutamine is necessary for the preservation of normal intestinal morphology and function in humans. The current study does not address this question in humans, but does provide information that demonstrates the detrimental effects of feeding a glutamine-free enteral diet and the inhibition of de novo synthesized glutamine in the infant rat intestine. The results described here support the hypothesis that both dietary and endogenously synthesized glutamine enhance morphologic indicators of intestinal integrity in infant rats. For both ethical and technical reasons, similar studies would be very difficult in humans, especially critically ill neonates, whose intestines are most likely to be susceptible to the effects of glutamine deprivation. It is also very difficult to accurately quantify villus and crypt dimensions and compare them from one patient to another in human punch biopsy material.

\section{CONCLUSION}

This study demonstrates that deprivation of dietary or endogenously synthesized glutamine results in a breakdown of the intestinal epithelium. We speculate that lack of glutamine in the diet and factors that decrease endogenous glutamine synthesis may result in deleterious consequences, which extend 
beyond the intestinal barrier, such as the development of systemic inflammatory response and alterations of the immune response.

\section{REFERENCES}

1. Ziegler TR, Young LS, Benfell K, Scheltinga M, Hortos K, Bye R, Morrow FD, Jacobs DO, Smith RJ, Antin JH 1992 Clinical and metabolic efficacy of glutaminesupplemented parenteral nutrition after bone marrow transplantation. Ann Intern Med 116:821-828

2. MacBurney M, Young LS, Ziegler TR, Wilmore DW 1994 A cost-evaluation of glutamine supplemented parenteral nutrition in adult bone marrow transplant patients. J Am Dietetic Assoc 94:1263-1266

3. Neu J, Roig JC, Meetze WH, Veerman M, Carter C, Millsaps M, Bowling D, Dallas MJ, Sleasman J, Knight T, Auestad N 1997 Enteral glutamine supplementation for very low birth weight infants decreases morbidity. J Pediatr 131:691-699

4. Dallas MJ, Bowling D, Roig JC, Auestad N, Neu J 1998 Enteral glutamine supplementation for very-low-birth-weight infants decreases hospital costs. JPEN J Parenter Enteral Nutr 22:352-356

5. Lacey JM, Crouch JB, Benfell K, Ringer SA, Wilmore CK, Maguire D, Wilmore DW 1996 The effects of glutamine-supplemented parenteral nutrition in premature infants. JPEN J Parenter Enteral Nutr 20:74-80

6. Suzuki I, Matsumoto Y, Adjei AA, Asato L, Shinjo S, Yamamoto S 1993 Effect of a glutamine-supplemented diet on response to methicillin-resistant Staphylococcus aureus infection in mice. J Nutr Sci Vitaminol (Tokyo) 39:405-410

7. Yoshida S, Yamasaki K, Kaibera A 1993 Glutamine supplementation in septic rats. Nippon Geka Gakki Zasshi 94:1078-1085

8. O'Dwyer ST, Smith RJ, Hwang TL, Wilmore DW 1989 Maintenance of small bowel mucosa with glutamine-enriched parenteral nutrition. JPEN J Parenter Enteral Nutr 13:579-85

9. Baskerville A, Hambleton P, Benbough JE 1980 Pathologic features of glutaminase toxicity. Br J Exp Pathol 61:132-138

10. Roig JC, Shenoy VB, Chakrabarti R, Lau YN, Neu J 1995 Localization of rat small intestine glutamine synthetase using immunofluorescence and in situ hybridization. JPEN J Parenter Enteral Nutr 19:179-181

11. Shenoy V, Roig JC, Chakrabarti R, Kubilis P, Neu J 1996 Ontogeny of glutamine synthetase in rat small intestine. Pediatr Res 39:643-648

12. Plauth M, Raible A, Vieillard-Baron D, Bauder-Gross D, Hartmann F 1999 Is glutamine essential for the maintenance of intestinal function? A study in the isolated perfused rat small intestine. Int J Colorectal Dis 14:86-94

13. Windmueller HG, Spaeth AE 1974 Uptake and metabolism of plasma glutamine by the small intestine. J Biol Chem 249:5070-5079

14. Purich DL 1998 Advances in the enzymology of glutamine synthetase. Adv Enzymol Relat Areas Mol Biol 72:9-42

15. DeMarco V, Dyess K, Strauss D, West CM, Neu J 1999 Inhibition of glutamine synthetase decreases proliferation of cultured rat intestinal epithelial cells. J Nutr 129:57-62

16. Weiss MD, DeMarco V, Strauss DM, Samuelson DA, Lane ME, Neu J 1999 Glutamine synthetase: a key enzyme for intestinal epithelial differentiation? JPEN J Parenter Enteral Nutr 23:140-146

17. Phillips AF, Anderson GG, Dvorak B, Williams CS, Lohe M, Lebourton V, Koldovsky O 1997 Growth of artificially fed infant rats: effect of supplementation with insulin-like growth factor 1. Am J Physiol 272:R1532-R1539

18. Auestad N, Korsak RA, Bergstrom JD, Edmond J 1989 Milk-substitutes comparable to rat's milk: their preparation, composition and impact on development and metabolism in the artificially-reared rat. Br J Nutr 61:495-518

19. Sheehan DC, Hrapchak BB 1987 Theory and Practice of Histotechnology, 2nd Ed. Battelle Press, Columbus, pp 59-65 and 143-144
20. Hayat MA 1989 Principles and Techniques of Electron Microscopy Biological Applications, 3rd Ed. CRC Press, Boca Raton, FL, pp 79, 326

21. Deitch EA, Winterton J, Ma L, Berg RD 1987 Gut as a portal of entry for bacteremia, the role of protein malnutrition. Ann Surg 205:681-692

22. Van Leeuwen PA, Boermeister MA, Houdijk AP, Ferwerda CC, Cuesta MA, Meyer S, Wesdorp RI 1994 Clinical significance of translocation. Gut 35(1 suppl):S28-S34

23. O’Boyle CJ, MacFie J, Mitchell CJ, Johnstone D, Sagar PM, Sedman PC 1998 Microbiology of bacterial translocation in humans. Gut 42:29-35

24. Souba WW, Klimberg S, Hautamaki RD, Mendenhall WH, Bova FC, Howard RJ, Bland KI, Copeland EM 1990 Oral glutamine reduces bacterial translocation following abdominal radiation J Surg Res 48:1-5

25. Gianotti L, Alexander JW, Gennari R, Pyles R, Babcock GF 1995 Oral glutamine decreases bacterial translocation and improves survival in experimental gut-origin sepsis. JPEN J Parenter Enteral Nutr 19:69-74

26. Chen K, Okuma T, Okamura K, Torigoe Y, Miyauchi Y 1994 Glutaminesupplemented parenteral nutrition improves gut mucosa integrity and function in endotoxemic rats. JPEN J Parenter Enteral Nutr 18:167-171

27. Klimberg VS, Salloum RM, Kasper M, Plumley DA, Dolson DJ, Hautamaki RD, Mendenhall WR, Bova FC, Bland KI, Copeland III EM 1990 Oral glutamine accelerates healing of the small intestine and improves outcome after whole abdominal radiation. Arch Surg 125:1049-1045

28. Li J, Langkamp-Henken B, Suzuki K, Stahlgren LH 1994 Glutamine prevents parenteral nutrition-induced increases in intestinal permeability. JPEN J Parenter Enteral Nutr 18:303-307

29. van der Hulst RR, van Kreel BK, von Meyenfeldt MF, Brummer RJ, Arends JW, Deutz NE, Soeters PB 1993 Glutamine and the preservation of gut integrity. Lancet 341:1363-1365

30. Panigrahi P, Gewolb IH, Bamford P, Horvath K 1997 Role of glutamine in bacterial transcytosis and epithelial cell injury. JPEN J Parenter Enteral Nutr 21:75-80

31. Kouznetsova L, Bijlsma PB, van Leeuwen PA, Groot JA, Houdijk AP 1999 Glutamine reduces phorbol-12,13-dibutyrate-induced macromolecular hyperpermeability in HT-29Cl.19A intestinal cells. JPEN J Parenter Enteral Nutr 23:136-139

32. Neu J, DeMarco VG, Gulig PA 2000 Bacterial translocation is exacerbated by inhibition of glutamine synthetase. Pediatr Res 47:293A(abstr)

33. Stoll B, Burrin DG, Henry J, Yu H, Jahoor F, Reeds PJ 1999 Substrate oxidation by the portal drained viscera of fed piglets. Am J Physiol 277:E168-E175

34. Windmueller HG 1982 Glutamine utilization by the small intestine. Adv Enzymol 53:202-237

35. Matthews CK, van Holde KE 1990 Biochemistry. Benjamin/Cummings Publishing, Redwood City, CA, pp 557-558

36. Rhoads JM, Argenzio RA, Chen W, Graves LM, Licato LL, Blikslager AT, Smith J, Gatzky J, Brenner DA 2000 Glutamine metabolism stimulates intestinal cell MAPKs by a cAMP inhibitable Raf independent mechanism. Gastroenterology 118:90-100

37. Blicklayer AT, Rhoads JM, Bristol DG, Roberts MC, Argenzio RA 1999 Glutamine and transforming growth factor alpha stimulate extracellular regulated kinases and enhance recovery of villous surface area in porcine ischemia injured intestine. Surgery 125:186-194

38. Ko YG, Kim EK, Kim T, Park H, Park HS, Choi EJ, Kim S 2001 Glutamine dependent antiapoptotic interaction of human glutaminyl-tRNA synthetase with apoptosis signal regulating kinase J Biol Chem 276:6030-6036

39. Beaulieu JF, Calvert R 1985 Permissive effect of glutamine on the differentiation of fetal mouse small intestine in organ culture. Differentiation 29:50-55

40. Oppenheimer SB, Edidin M, Orr CW, Roseman S 1969 An L-glutamine requirement for intercellular adhesion. Proc Natl Acad Sci USA 63:1395-1402

41. Khan J, Iiboshi Y, Cui L, Wasa M, Sando K, Tagaki Y, Okada A 1999 Alanylglutamine supplemented parenteral nutrition increases luminal mucus gel and decreases permeability in the rat small intestine JPEN J Parenter Enteral Nutr 23:24-31

42. Buchman AL 2001 Glutamine commercially essential or conditionally essential? A critical appraisal of human data. Am J Clin Nutr 74:25-32 